\title{
Composição lipídica de carne bovina de grupos genéticos taurinos e zebuínos terminados em confinamento ${ }^{1}$
}

\author{
Lizandra Vercezi Rossatoㄹ, Maria Cristina Bressan², Érika Cristina Rodrigues ${ }^{2}$, Maria Inês Alves \\ de Carvalho Martins Carolino ${ }^{3}$, Rui José Branquinho Bessa ${ }^{3}$, Susana Paula Pereira Alves ${ }^{3}$ \\ ${ }^{1}$ Trabalho financiado pela CAPES e pelo CNPq. \\ 2 Universidade Federal de Lavras, Departamento Ciência dos Alimentos, Caixa Postal 3037 - 37200-000, Lavras, MG - Brasil. \\ ${ }^{3}$ Estação Zootécnica Nacional - INRB, Fonte Boa-2005-048, Vale de Santarém - Portugal.
}

RESUMO - O objetivo neste trabalho foi avaliar a composição em lipídeos totais, colesterol e ácidos graxos do músculo longissimus dorsi de bovinos Bos taurus $(\mathrm{n}=40)$ e Bos indicus $(\mathrm{n}=40)$ com 30 a 36 meses de idade e peso de carcaça entre 240 e $270 \mathrm{~kg}$ terminados em confinamento por 90 dias. Os resultados de lipídeos totais foram semelhantes entre os dois grupos. O nível de colesterol foi mais elevado em Bos indicus (66,95 mg/100 g) que em Bos taurus (37,37 mg/100 g). Os valores médios dos ácidos graxos saturados de C14:0 e C18:0 foram mais elevados nos Bos indicus (3,52 e 22,45\%) em comparação aos Bos taurus (3,08 e 18,67\%). O C16:0 nos Bos taurus (26,14\%) foi maior que nos Bos indicus (24,77\%). $\mathrm{Na}$ análise do perfil de ácidos graxos monoinsaturados (AGM), os Bos taurus apresentaram os percentuais mais elevados de C14:1 cis9, C16:1 cis9, C18:1 cis9, ¿AGM e os menores de C18:1 trans. Os animais Bos taurus apresentaram maiores percentuais

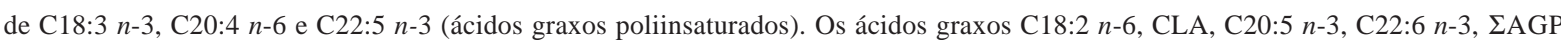
e $\Sigma n-6$ foram semelhantes entre grupos de bovinos. A razão $n-6 / n-3$ foi menor em Bos taurus $(4,63)$ em comparação aos Bos indicus (5,70). Em geral, o grupo genético influencia o perfil de colesterol e ácidos graxos de bovinos e esse efeito é mais pronunciado nos ácidos graxos saturados e monoinsaturados.

Palavras-chave: ácidos graxos, colesterol, suplementação

\section{Lipid composition of meat from zebu and taurine cattle finished in confinement}

\begin{abstract}
This study was carried out to evaluate total lipids (TL), cholesterol and fatty acids (FA) in the longissimus dorsi muscle of Bos taurus $(\mathrm{n}=40)$ and Bos indicus $(\mathrm{n}=40)$ bulls, aged 30-36 months and 240-270 kg carcass weight, finished in the feedlot for 90 days. The total lipids content was similar in both groups. Cholesterol was higher in Bos indicus (66.95 mg/100 g) than in Bos taurus (37.37 mg/100 g). For saturated fatty acids, the means for C14:0 and C18:0 were higher in Bos indicus (3.52 and 22.45\%), than in Bos taurus (3.08 e 18.67\%). The C16:0 was higher in Bos taurus (26.14\%), as compared to Bos indicus (24.77\%). For monounsaturated fatty acids (MUFA), Bos taurus had higher levels of C14:1 cis9, C16:1 cis9, C18:1 cis9, इMUFA but lower levels of C:18:1 trans. For polyunsaturated fatty acids (PUFA), Bos taurus presented higher percentages of C18:3 n-3, C20:4 n-6 and C22:5 n-3. The C18:2 n-6, CLA,

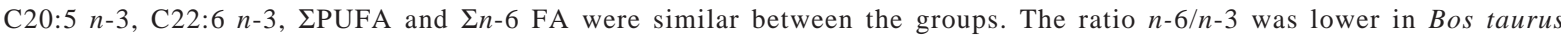
(4.63) than in Bos indicus (5.70). In general, there was a genetic influences in the cholesterol and fatty acids of cattle profile, and this effect was more pronounced for saturated and monounsaturated fatty acids.
\end{abstract}

Key Words: cholesterol, fatty acids, supplementation

\section{Introdução}

O Brasil é atualmente o maior exportador de carne bovina com um rebanho comercial estimado em 207,2 milhões de animais (IBGE, 2007). Nesse rebanho, 80\% dos animais possuem genes Bos indicus na forma de animais puros ou cruzados que estão distribuídos no território brasileiro (Mariante et al., 1982). Nas regiões mais quentes, as raças
Bos indicus prevalecem, em decorrência de sua maior adaptação, enquanto os Bos taurus são mais utilizados nas regiões de clima ameno. Assim, considerando as dimensões e a variabilidade genética, é possível que a qualidade nutricional da carne brasileira apresente variações. Além disso, as informações encontradas na literatura para o efeito genético no perfil lipídico foram obtidas a partir de estudos entre raças taurinas ou entre raças zebuínas, 
entretanto, comparações entre zebuínos e taurinos são raras ou não existem.

A composição dos ácidos graxos da gordura da carne de bovinos, por suas implicações na saúde humana, tem recebido considerável interesse (Nuernberg et al., 2005). Grandes mudanças no perfil lipídico podem ser introduzidas alterando a alimentação, entretanto, os fatores genéticos são investigados em menor escala, embora muitos estudos reportem diferenças na composição de ácidos graxos em diferentes espécies animais atribuídas à raça (De Smet, 2004).

O teor de lipídeos, colesterol e a proporção dos ácidos graxos saturados (AGS) e ácidos graxos trans na alimentação humana são considerados fatores de risco na ocorrência de doenças cardiovasculares (Scollan et al., 2001). Por outro lado, os ácidos graxos poliinsaturados (AGP) oriundos do C18:2n-6 e C18:3n-3 parecem oferecer proteção contra essas doenças (Williams, 2000) e a razão $n-6 / n-3$ recomendada para uma dieta saudável deve ser menor que 4:1 (Committee of Medical Aspects of Food Policy, 1994). Os ácidos graxos poliinsaturados, além dos n-6 e $n-3$, incluem isômeros conjugados do ácido linoleico (CLA) que apresentam atividade imunoestimulatória, antimutagênica e antioxidante, independentemente da quantidade e do tipo de gordura consumida (Ip, 1997).

O objetivo neste trabalho foi avaliar as características lipídicas (lipídeos totais, colesterol e ácidos graxos) do músculo longissimus dorsi de bovinos de diferentes grupos genéticos (Bos indicus e Bos taurus) terminados em confinamento.

\section{Material e Métodos}

Foram utilizados animais de origem Bos indicus (Tabapuã, n=20 e Gir, n=20) e Bos taurus (Holandês, n=20 e Simental, n=20), machos não-castrados com 30 a 36 meses de idade, peso de carcaça entre 240 e 270 kg e mínimo de 3 mm de gordura subcutânea, terminados em sistema de confinamento por 90 dias antes do abate. Os animais foram mantidos durante o confinamento com dieta composta de $50 \%$ de volumoso (cana-de-açúcar) e 50\% de concentrado (milho grão, farelo de soja e ureia) e foram abatidos segundo as normas legais, em matadouro-frigorífico localizado no estado de São Paulo em outubro de 2006.

As amostras do músculo longissimus dorsi foram coletadas na região entre a $5 \underline{a}$ e $7 \underline{a}$ costelas 24 horas post mortem. As peças foram embaladas em papel alumínio, identificadas, congeladas em túnel de congelamento $\left(-35^{\circ} \mathrm{C}\right)$ e armazenadas a $-18^{\circ} \mathrm{C}$. As análises de lipídeos totais, extração de lipídeos e colesterol foram realizadas no
Laboratório de Tecnologia de Carnes do Departamento Ciência dos Alimentos da Universidade Federal de Lavras, Minas Gerais, de novembro de 2006 a março de 2007. A determinação dos ácidos graxos foi feita no Laboratório de Metabolismo Lipídico da Estação Zootécnica Nacional, Santarém, Portugal, no período de março a maio de 2007.

Os músculos longissimus dorsi foram descongelados a $4^{\circ} \mathrm{C}$ e submetidos à retirada da gordura. Os lipídeos totais foram determinados segundo AOAC (1995) e expressos em percentuais da matéria integral. Para determinação dos ácidos graxos e colesterol, $5 \mathrm{~g}$ de amostra foram submetidos à extração de lipídeos, segundo Folch et al. (1957), utilizando-se clorofórmio metanol 2:1 (vol:vol). A determinação do colesterol foi realizada por colorimetria, de acordo com Bohac et al. (1988), com adaptações de Bragagnolo \& Rodriguez-Amaya (2002). As análises de lipídeos totais e colesterol foram realizadas em duplicata.

A determinação dos ácidos graxos seguiu os protocolos de Hartman \& Lago (1973), procedendo-se à saponificação com solução de hidróxido de sódio em metanol $0.5 \mathrm{M}$, seguida de metilação com cloreto de amônia, metanol e ácido sulfúrico. Posteriormente, os extratos foram submetidos a cromatografia gasosa, em cromatógrafo HP 6890 (Agilent Technologies Inc., Palo Alto, CA, USA), equipado com detector de ionização de chama, injetor split na razão 1:50 e coluna capilar da CP-Sil 88,100 m × $0.25 \mathrm{~mm}$ $\times 0.20 \mu \mathrm{m}$ (Varian Inc., Walnut Creek, CA, USA) e os sinais processados pelo software Agilent Chemstation (Agilent Technologies Inc., Palo Alto, CA, USA). As condições cromatográficas foram temperatura inicial da coluna de $100^{\circ} \mathrm{C} / 15$ minutos; aumentada $10^{\circ} \mathrm{C} /$ minuto até $150^{\circ} \mathrm{C}$ e mantida por 5 minutos; depois a $1^{\circ} \mathrm{C} /$ minuto até os $158^{\circ} \mathrm{C} \mathrm{e}$ mantida por 30 minutos; e novamente a $1^{\circ} \mathrm{C} /$ minuto até $200^{\circ} \mathrm{C}$ e mantida por 60 minutos. A temperatura do injetor foi $250^{\circ} \mathrm{C}$ e a do detector $280^{\circ} \mathrm{C}$. O gás de arraste utilizado foi o hélio a pressão constante de 32,78 psi. Os ácidos graxos foram identificados por comparação aos tempos de retenção apresentados pelo padrão cromatográfico de C4:0 a C24:0 (Supelco ${ }^{\mathrm{TM}} 37$ standard FAME Mix, Supelco Inc., Bellefonte, PA, USA) e expressos em porcentagem do total de ácidos graxos identificados.

O delineamento utilizado no experimento foi inteiramente casualizado, com animais puros oriundos dos grupos genéticos Bos taurus e Bos indicus, considerando cada animal uma unidade experimental. Os dados obtidos foram submetidos a análise de variância e ao teste $\mathrm{F}$ a 5\% de significância utilizando-se o programa computacional SISVAR(Ferreira, 1992). 


\section{Resultados e Discussão}

A composição em lipídeos totais foi semelhante entre as amostras de bovinos Bos taurus e Bos indicus (Tabela 1). Alguns autores, como Laborde et al. (2001), em animais Simental e Red Angus, e Nuernberg et al. (2005), em animais Holstein e Simental, observaram ausência do efeito da raça no teor de lipídeos totais em Bos taurus terminados com suplementação de grãos. Outros autores verificaram em Bos indicus maior porcentagem de gordura que em Bos taurus. Moreira et al. (2003) observaram em Nelore média mais elevada de lipídeos totais que em Nelore $\times$ Limousin e Vaz et al. (2001) notaram que, quanto maior a participação de sangue de Bos indicus na formação do grupo genético, maiores os percentuais de lipídeos totais.

Normalmente, as taxas de lipídeos totais observadas em bovinos comerciais variam de 1 a 3\% na Bélgica (Cuvelier et al., 2006), na Itália (Cifuni et al., 2004), em Portugal (Alfaia et al., 2007) e na Espanha (Serra et al., 2008); e de 4 a 5\% na Argentina (Garcia et al., 2008) e Nova Zelândia (Purchas \& Zou, 2008) e acima de 10\% no Japão (Purchas
\& Zou, 2008). Neste trabalho, as taxas de gordura no longissimus dorsi foram superiores à maioria dos valores relatados na literatura. Isso, no perfil lipídico, implica em geral aumento na proporção de ácidos graxos saturados e redução nos ácidos graxos poliinsaturados (Cordain et al., 2002).

A média de colesterol foi significativamente mais elevada $(\mathrm{P}<0,05)$ em Bos indicus que em Bos taurus. Entretanto, Moreira et al. (2003) não encontraram diferença entre animais Bos indicus (35,16 mg/100 g) e animais cruzados (39,64 mg/100 g), assim como Wheeler et al. (1987), em bovinos Chianina e Hereford $\times$ Angus (com médias de 62,71 e 63,92 mg/100 g, respectivamente).

Houve efeito $(\mathrm{P}<0,05)$ do grupo genético sobre o perfil de ácidos graxos saturados, pois os resultados de C14:0 e C18:0 foram mais altos nos animais Bos indicus. Entretanto, animais Bos taurus apresentaram percentuais mais elevados de C16:0 em comparação aos Bos indicus. Segundo Woollett et al. (1992), os ácidos C16:0 e C14:0 enriquecem os fosfolipídeos das membranas celulares, interferindo na função normal dos receptores de lipoproteínas de baixa densidade (LDL), reduzindo sua remoção e

Tabela 1 - Composição em lipídeos totais, colesterol e ácidos graxos ${ }^{\mathrm{a}}$ no músculo Longissimus dorsi de bovinos

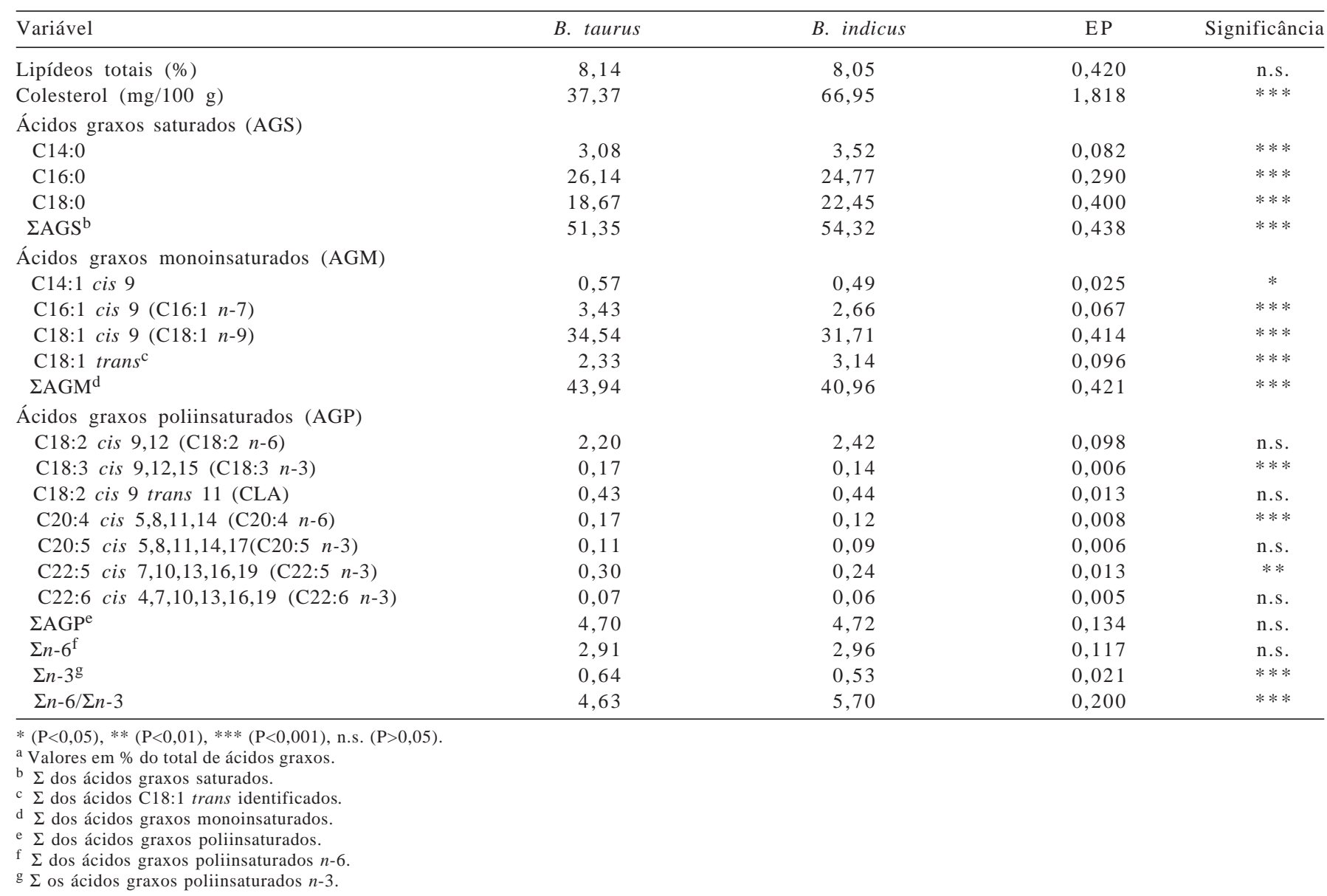


aumentando sua concentração no plasma, portanto, são considerados hipercolesterolêmico. Entre esses, o C14:0 é o mais hipercolesterolêmico e possui potencial para elevar a concentração plasmática de colesterol quatro vezes mais que o C16:0.

Animais Bos taurus apresentaram médias mais baixas no $\Sigma$ AGS em comparação aos Bos indicus. Valores médios mais baixos foram observados por Rodrigues et al. (2004) em animais Bos indicus das raças Nelore (42,1\%) e mestiços $1 / 2$ Nelore $\times 1 \frac{1}{2}$ Sindhi $(39,8 \%$ ) e por Laborde et al. (2001) em bovinos Simental (44,13\%) e Red Angus (46,22\%). Entretanto, em animais Charolês, Nelore e mestiços confinados recebendo concentrado, Menezes et al. (2006) encontraram médias de 50,2\% a 51,7\% de ácidos graxos saturados totais.

Em comparação aos Bos indicus, os animais Bos taurus apresentaram os maiores percentuais de ácidos graxos monoinsaturados (AGM), de C14:1 cis9, C16:1 cis9 e C18:1cis9. Segundo Feldman (2002), os ácidos graxos monoinsaturados têm efeitos na redução de doenças cardiovasculares por reduzirem as concentrações plasmáticas de LDL (lipoproteínas de baixa densidade), além de inibirem a agregação plaquetária.

As diferenças nas concentrações de C14:0, C18:0, C14: 1 cis9 e C18:1 cis9 consideradas em conjunto sugerem que a atividade da enzima delta-9 dessaturase foi mais elevada no grupo Bos taurus que no grupo Bos indicus. Essa enzima introduz uma dupla ligação na posição 9 a contar do grupo carboxílico e de geometria cis em AGS (ou trans monoinsaturados). Diferença entre genótipos quanto à expressão da delta-9 dessaturase foi reportada por Laborde et al. (2001) e Taniguchi et al. (2004).

A média encontrada para o total de ácidos graxos C18:1 trans foi mais elevada $(\mathrm{P}<0,05)$ em animais Bos indicus que em Bos taurus. O total de ácidos graxos C18:1 trans é formado, em sua maioria, pelo ácido vacênico (C18:1 trans11), produto da biohidrogenação do C18:2 n-6. O ácido vacênico, no tecido adiposo, é convertido em ácido linoleico conjugado (CLA) por ação da delta-9 dessaturase (Scollan et al., 2006). Salminen et al. (1998) estudaram o consumo de C18:1 trans 11 durante cinco semanas no homem e observaram que o nível sérico de ácido linoleico conjugado aumentou e que o C18:1 trans11 afetou positivamente a síntese endógena de ácidos linoleicos conjugados. Em modelos animais, o CLA tem proprie-dades anticarcinogênicas, antiaterogênicas e modulador das respostas imunes (Whigham et al., 2000; Belury, 2002). Entretanto, os isômeros CLA mostram atividades biológicas diferentes: o trans 10,cis12 tem ação no metabolismo lipídico; enquanto o cis9,trans11 e o trans10,cis12 mostram efeito anticarcinogênico (Pariza et al., 2001; Evans et al., 2002).

Animais Bos taurus apresentaram percentuais mais elevados de AGP C18:3 n-3, C20:4 n-6 e C22:5n-3 em comparação aos Bos indicus. Entretanto, os ácidos graxos C18:2 n-6; CLA; C20:5 n-3 e C22:6 n-3 foram semelhantes entre os grupos. O $\Sigma$ AGP também foi semelhante entre Bos taurus e Bos indicus. O consumo de ácidos graxos poliinsaturados é importante, pois esses ácidos graxos reduzem os níveis séricos de colesterol; alguns são considerados essenciais (não são sintetizados pelo organismo) e são precursores de várias substâncias, algumas vasoativas, que influenciam a viscosidade sanguínea, a permeabilidade dos vasos e a pressão arterial. O aumento de alguns desses ácidos graxos (n-6), ou a alteração da razão entre eles ( $n-6 / n$-3), pode aumentar a produção de tromboxanos e leucotrienos, que, em excesso, estão associados a doenças como tromboses, arritmias, artrite, asma e psoríase (Belda \& Pourchet-Campos, 1991).

O $\Sigma$ AGP e o $\Sigma$ AGP da série $n-6$ foi semelhante entre Bos taurus e Bos indicus. Entretanto, a concentração do $\Sigma$ AGP da série $n-3$ foi maior em animais Bos taurus que em Bos indicus. Dessa forma, a relação $n-6 / n-3$ foi mais baixa em Bos taurus que em Bos indicus. A influência das raças do grupo Bos taurus na razão $\Sigma n-6 / \Sigma n-3$ foi reportada por Laborde et al. (2001) em estudo com animais Simental $(4,42)$ e Red Angus $(3,54)$ e por Nuernberg et al. (2005) em animais Holstein $(6,49)$ e Simmental $(8,34)$. Entre raças do grupo Bos indicus, foram descritas diferenças entre raças por Rodrigues et al. (2004) em animais cruzados $1 \frac{1}{2}$ Nelore $\times 1 \frac{1}{2} \operatorname{Sindi}(8,80) \times$ Nelore $(6,08)$. Entretanto, os valores dessa relação $n-6 / n-3$ foram mais baixos, com variações de 1,69 a 2,22 (Rule et al., 2002; Nuernberg et al., 2005; Padre et al., 2006).

Nos dados gerais, os bovinos Bos indicus apresentaram percentual mais elevado de $\Sigma$ AGS, superior aos Bos taurus em 2,97\%. Entretanto, grande parte dessa diferença é atribuída ao percentual de C18:0, que foi superior nos Bos indicus, em relação aos Bos taurus. Esse ácido graxo (C18:0) não é relacionado à elevação sérica do colesterol ou problemas cardiovasculares, pois, uma vez no organismo, sofre modificações e passa a C18:1 cis-9 (Motta, 2005).

Os resultados médios de $\Sigma n$-3 neste trabalho foram relativamente baixos, assim como a diferença observada entre Bos taurus e Bos indicus (0,11\%). Por outro lado, em animais terminados em pastagens, valores mais elevados no $\Sigma n$-3, variando de 1,27 a 4,70\%, forma observados por Rule et al. (2002), Nuernberg et al. (2005) e Padre et al. (2006).

Os grupos genéticos Bos taurus e Bos indicus apresentam diferenças nos percentuais de colesterol e no 
perfil de ácidos graxos. Nos ácidos graxos, as diferenças observadas entre genótipos são relativamente pequenas se comparadas às diferenças observadas entre animais terminados com concentrados ou pasto (Rule et al., 2002; Nuernberg et al., 2005). Assim, em animais terminados com concentrados, o fator genético teve efeito mais pronunciado nos ácidos graxos saturados e monoinsaturados.

\section{Conclusões}

Em animais Bos taurus e Bos indicus terminados no sistema de confinamento, o grupo genético influencia as taxas de colesterol e a composição de ácidos graxos do músculo longissimus dorsi, de forma que efeitos mais pronunciados são encontrados nos ácidos graxos saturados e monoinsaturados.

\section{Agradecimentos}

À CAPES, ao CNPq e à FAPEMIG, pelo apoio na forma de bolsas.

Ao Serviço de Inspeção Federal, pelo auxílio na obtenção e conservação das amostras, e à Empresa Minerva S.A., pela doação das amostras.

Aos professores Eduardo Mendes Ramos, do DCA/ UFLA, e Luís Telo da Gama, da EZN/INRB, e aos alunos de Pós-graduação Sandra Aparecida Tavares e Rafael Vilhena Reis Neto, pelo apoio na condução do trabalho.

\section{Literatura Citada}

ALFAIA, C.M.M.; CASTRO, M.L.F.; MARTINS, S.I. et al. Effect of slaughter season on fatty acid composition, conjugated linoleic acid isomers and nutritional value of intramuscular fat in Barrosã-PDO veal. Meat Science, v.75, n.1, p.44-52, 2007.

ASSOCIATION OF OFFICIAL ANALYTICAL CHEMISTS - AOAC. Official Methods of Analysis of the Association of Official Analytical Chemists. 16.ed. Arlington: AOAC, 1995.

BELDA, M.C.R.; POURCHET-CAMPOS, M.A. Ácidos graxos essenciais em nutrição: uma visão atualizada. Ciência e Tecnologia de Alimentos, v.11, n.1, p.5-35, 1991.

BELURY, M.A. Dietary conjugated linoleic acid in health: physiological effects and mechanisms of action. Annual Review of Nutrition, v.22, p.505-531, 2002.

BOHAC, C.E.; RHEE, K.S.; CROSS, H.R. et al. Assessment of methodologies for colorimetric cholesterol assay of meats. Journal Food Science, v.53, n.6, p.1642-1645, 1988.

BRAGAGNOLO, N.; RODRIGUEZ-AMAYA, D.B. Teores de colesterol, lipídeos totais e ácidos graxos em cortes de carne suína. Ciência e Tecnologia de Alimentos, v.22, n.1, p.1-13, 2002.

CIFUNI, G.F.; NAPOLITANO, F.; RIVIEZZI, A.M. et al. Fatty acid profile, cholesterol content and tenderness of meat from Podolian young bulls. Meat Science, v.67, n.2, p.289-297, 2004.

COMMITTEE OF MEDICAL ASPECTS OF FOOD POLICY. Nutritional aspects of cardiovascular disease. London:
Department of Healthy, HMSO, 1994. (Report on Healthy and Social Subjects, 46).

CORDAIN, L.; WATKINS, B.A.; FLORANT, G.L. et al. Fatty acid analysis of wild ruminant tissues: evolutionary implications for reducing diet-related chronic disease. European Journal of Clinical Nutrition, v.56, n.3, p.181-191, 2002.

CUVELIER, C.; CABARAUX, J.F.; DUFRASNE, I. et al. Comparison of composition and quality traits of meat from young finishing bulls from Belgian Blue, Limousin and Aberdeen Angus breeds. Meat Science, v.74, n.3, p.522-531, 2006.

DE SMET, S.; RAES, K.; DEMEYER, D. Meat fatty acid composition as affected by fatness and genetic factors: a review. Animal Research, v.53, p.81-98, 2004.

EVANS, M.; BROWN, J.; MCINTOSH, M. Isomer-specific effects of conjugated linoleic acid (CLA) on adiposity and lipid metabolism. Journal of Nutritional Biochemistry, v.13, p.508-512, 2002.

FELDMAN, E.B. The scientific evidence for a beneficial health relationship between walnuts and coronary heart disease. The Journal of Nutrition, v.132, n.5, p.1062-1101, 2002.

FERREIRA, D.F. SISVAR (Sistema para Análise de Variância para Dados Balanceados). Lavras: Universidade Federal de Lavras, 1992. 79p.

FOLCH, J.; LESS, M.; STANLEY, S.A. simple method for the isolation and purification of total lipids from animal tissues. Journal of Biological Chemistry, v.226, n.1, p.497-509, 1957.

GARCIA, P.T.; PENSEL, N.A.; SANCHO, A.M. et al. Beef lipids in relation to animal breed and nutrition in Argentina. Meat Science, v.79, n.3, p.500-508, 2008.

HARTMAN, L.; LAGO, R.C. Rapid preparation of fatty acid methyl esters from lipids. Laboratory Pratice, v.22, p.475-476, 1973.

INSTITUTO BRASILEIRO DE GEOGRAFIA E ESTATÍSTICA IBGE. [2007]. Produção da pecuária. Disponível em: <http:// www.ibge.gov.br/home/presidencia/noticias/noticia_visualiza.php? id_notícia=759\&id_página=1>. Acesso em: 15/10/2007.

IP, C. Review of the effects of trans fatty acids, oleic acid, n-3 polyunsaturated fatty acids, and conjugated linoleic acid on mammary carcinogenesis in animals. American Journal of Clinical Nutrition, v.66, n.6, p.1523-1529, 1997.

LABORDE, F.L.; MANDELL, I.B.; TOSH, J.J. et al. Breed effects on growth performance, carcass characteristics, fatty acid composition, and palatability attributes in finishing steers. Journal of Animal Science, v.79, n.2, p.355-365, 2001.

MARIANTE, A.S.; FIGUEIREDO, G.R.; ROSA, A.N. et al. Efeito de grupos genéticos sobre características de carcaças bovinas. In: REUNIÃO ANUAL DA SOCIEDADE BRASILEIRA DE ZOOTECNIA, 19., 1982, Piracicaba. Anais... Piracicaba: Sociedade Brasileira de Zootecnia, 1982. p.233-234. (CD-ROM)

MENEZES, L.F.G.; KOZLOSKI, G.V.; DESCHAMPS, F.C. et al. Perfil de ácidos graxos de cadeia longa e qualidade da carne de novilhos terminados em confinamento com diferentes níveis de monensina sódica na dieta. Ciência Rural, v.36, n.1, p.186-190, 2006.

MOREIRA, F.B.; SOUZA, N.E.; MATSUSHITA, M. et al. Evaluation of carcass characteristics and meat chemical composition of Bos indicus and Bos indicus x Bos taurus crossbred steers finished in pasture systems. Brazilian Archives of Biology and Technology, v.46, n.4, p.607-614, 2003.

MOTTA, V.T. Bioquímica. Caxias do Sul: Educs, 2005. 332p.

NUERNBERG, K.; DANNENBERGER, D.; NUERNBERG, G. et al. Effect of a grass-based and a concentrate feeding system on meat quality characteristics and fatty acid composition of different cattle breeds. Livestock Production Science, v.94, n.1-2, p.137-147, 2005.

PADRE, R.G.; ARICETTI, J.A.; MOREIRA, F.B. et al. Fatty acid profile, and chemical composition of Longissimus muscle of bovine steers and bulls finished in pasture system. Meat Science, v.74, n.2, p.242-248, 2006. 
PARIZA, M.W.; PARK, Y.; COOK, M.E. The biologically active isomers of conjugated linoleic acid. Progress in Lipid Research, v.40, p.283-298, 2001.

PURCHAS, R.W.; ZOU, M. Composition and quality differences between the longissimus and infraspinatus muscles for several groups of pasture-finished cattle. Meat Science, v.80, n.2, p.470-479, 2008.

RODRIGUES, V.C.; BRESSAN, M.C.; CARDOSO, M.G. et al. Ácidos graxos na carne de búfalos e bovinos castrados e inteiros. Revista Brasileira de Zootecnia, v.33, n.2, p.434-443, 2004.

RULE, D.C.; BROUGHTON, K.S.; SHELLITO, S.M. et al. Comparison of muscle fatty acid profiles and cholesterol concentrations of bison, beef cattle, elk and chicken. Journal of Animal Science, v.80, p.1202-1211, 2002.

SALMINEN, I.; MUTANEN, M.; JAUHIANEN, M. et al. Dietary trans fatty acid increased conjugated linoleic acid levels in human serum. Nutrition Biochemistry, v.9, n.2, p.93-98, 1998.

SCOLLAN, N.D.; CHOI, N.J.; KURT, E. et al. Manipulating the fatty acid composition of muscle and adipose tissue in beef cattle. British Journal of Nutrition, v.85, p.115-124, 2001.

SCOLLAN, N.D.; HOCQUETTE, J.F.; NUERNBERG, K. et al. Innovations in beef production systems that enhance the nutritional and health value of beef lipids and their relationship with meat quality. Meat Science, v.74, n.1, p.17-33, 2006.
SERRA, X.; GUERRERO, L.; GUARDIA, M.D. et al. Eating quality of young bulls from three Spanish beef breed-production systems and its relationships with chemical and instrumental meat quality. Meat Science, v.79, n.1, p.98-104, 2008.

TANIGUCHI, M.; MANNEN, H.; SHIMAKURA, Y. et al. Differences in Stearoyl-CoA desaturase mRNA levels between Japanese Black and Holstein cattle. Livestock Production Science, v.87, n.2-3, p.215-220, 2004.

VAZ, F.N.; RESTLE, J.; FEIJÓ, G.L.D. et al. Quality and composition of meat from entire or castrated beef cattle from different Charolais x Nelore genetic groups. Revista Brasileira de Zootecnia, v.30, n.2, p.518-525, 2001.

WHEELER, T.L.; DAVIS, G.W.; STOECKER, B.J. et al. Cholesterol concentration of longissimus muscle, subcutaneous fat and serum of two beef cattle breed types. Journal of Animal Science, v.65, n.6, p.1531-1537, 1987.

WHIGHAM, L.; COOK, M.E.; ATKINSON, R.L. Conjugated linoleic acid: implications for human health. Pharmacological Research, v.42, n.6, p.503-510, 2000.

WILLIAMS, C.M. Dietary fatty acids and human health. Annales Zootechnie, v.49, p.165-180, 2000.

WOOLLETT, A.L.; SPADY, K.D.; DIETSCHY, M.J. Saturated and unsaturated fatty acids independently regulate low-density lipoprotein receptor activity and production rate. Journal of Lipid Research, v.33, n.1, p.77-88, 1992. 